\title{
Fully automatic cardiac segmentation from 3D CTA data: a multi-atlas based approach
}

\author{
Hortense A. Kirisli ${ }^{a}$, Michiel Schaap ${ }^{a}$, Stefan Klein $^{a}$, Lisan A. Neefjes ${ }^{b}$, Annick C. Weustink ${ }^{b}$, Theo \\ van Walsum ${ }^{a}$ and Wiro J. Niessen ${ }^{a, c}$ \\ ${ }^{a}$ Biomedical Imaging Group Rotterdam, Dept. of Radiology \& Medical Informatics, Erasmus MC, \\ Rotterdam, The Netherlands \\ ${ }^{b}$ Dept. of Radiology, Erasmus MC, Rotterdam, The Netherlands \\ ${ }^{c}$ Imaging Science \& Technology, Faculty of Applied Sciences, Delft Univ. of Technology, \\ Delft, The Netherlands
}

\begin{abstract}
Computed tomography angiography (CTA), a non-invasive imaging technique, is becoming increasingly popular for cardiac examination, mainly due to its superior spatial resolution compared to MRI. This imaging modality is currently widely used for the diagnosis of coronary artery disease (CAD) but it is not commonly used for the diagnosis of ventricular and atrial function. In this paper, we present a fully automatic method for segmenting the whole heart (i.e. the outer surface of the myocardium) and cardiac chambers from CTA datasets. Cardiac chamber segmentation is particularly valuable for the extraction of ventricular and atrial functional information, such as stroke volume and ejection fraction. With our approach, we aim to improve the diagnosis of CAD by providing functional information extracted from the same CTA data, thus not requiring additional scanning. In addition, the whole heart segmentation method we propose can be used for visualization of the coronary arteries and for obtaining a region of interest for subsequent segmentation of the coronaries, ventricles and atria. Our approach is based on multi-atlas segmentation, and performed within a non-rigid registration framework. A leave-one-out quantitative validation was carried out on 8 images. The method showed a high accuracy, which is reflected in both a mean segmentation error of $1.05 \pm 1.30 \mathrm{~mm}$ and an average Dice coefficient of 0.93 . The robustness of the method is demonstrated by successfully applying the method to 243 additional datasets, without any significant failure.
\end{abstract}

Keywords: cardiac segmentation, Computed Tomography Angiography (CTA), registration, multi-atlas based segmentation

\section{INTRODUCTION}

Assessment of ventricular and atrial function is valuable for cardiovascular diagnosis. This has led to the development of various automatic cardiac image segmentation methods. Most of these studies focused on the left ventricle (LV), because functional parameters derived from the diastolic and systolic LV volume are important predictors for heart disease ${ }^{1}$. More recently, researchers have addressed the problem of automatic segmentation of the four cardiac chambers ${ }^{234}$ which allows for the diagnosis of diseases in other chambers than the left ventricle, such as left atrial fibrillation and right ventricle (RV) overload. Whole heart (i.e. the outer surface of the myocardium) isolation ${ }^{5}$ is especially useful for the visualization of the coronary arteries and for obtaining a region of interest for subsequent segmentation of the coronaries, ventricles and atria.

Cardiac segmentation methods have been developed for a variety of cardiac imaging modalities, such as Computed Tomography Angiography (CTA), Magnetic Resonance Imaging (MRI) and Single Photon Emission Computed Tomography (SPECT). The proposed methods incorporate various techniques, such as model based segmentation ${ }^{36}$, atlas-based segmentation $^{7}$ and machine learning ${ }^{4}$.

This paper has two main contributions. First, we present a fully automatic method for segmenting the whole heart and cardiac chambers from 3D CTA datasets. CTA is a non-invasive imaging technique that is becoming increasingly popular for cardiac examination, mainly due to its superior spatial resolution compared to MRI. This imaging modality is currently widely used for the diagnosis of coronary artery disease (CAD) but it is not commonly used for the diagnosis of ventricular

Further author information:

H.A.Kirisli: E-mail: h.kirisli@erasmusmc.nl, Telephone: +31-10-7044246, Fax: +31-10-7044722

Medical Imaging 2010: Image Processing, edited by Benoit M. Dawant, David R. Haynor,

Proc. of SPIE Vol. 7623, 762305 - C 2010 SPIE · CCC code: 1605-7422/10/\$18 - doi: 10.1117/12.838370

Proc. of SPIE Vol. 7623 762305-1 
and atrial function ${ }^{1}$. We aim to improve the diagnosis of CAD by providing additional functional information, using conventional CTA imaging data, thus without additional imaging. Our approach is based on multi-atlas segmentation, and performed within a non-rigid registration framework. This technique is easy to use, and implementations are publicly available.

Second, we evaluate the method on a large number of datasets. The accuracy of our method is assessed with a quantitative evaluation on 8 patient cardiac CTA datasets and the robustness of the method is demonstrated with a qualitative evaluation on 243 cardiac CTA datasets.

In Section 2, we describe both the multi-atlas non-rigid registration technique and the atlas based segmentation method. The CTA acquisition protocol and a definition of the cardiac structures to be segmented are given in Section 3 . The quantitative and qualitative evaluation of our approach is presented in Section 4. The results and possible improvements are discussed in Section 5.

\section{METHOD}

\subsection{Registration : principle}

Registration aims to find a spatial alignment between a moving image $I_{M}(\mathbf{x})$ and a fixed image $I_{F}(\mathbf{x})$. This is achieved by applying a transformation $T(\mathbf{x})$ to $I_{M}(\mathbf{x})$. Mathematically, the registration process is expressed as an optimization problem:

$$
\widehat{T}=\arg \min _{T} \mathcal{C}\left(I_{F}(\mathbf{x}), I_{M}(T(\mathbf{x}))\right)
$$

where $\mathbf{x}$ is an element of $\Omega_{F}$, the fixed image domain, $\widehat{T}$ is the optimal transformation and $\mathcal{C}$ a cost function that measures the dissimilarity between the two images.

Different aspects need to be addressed when using registration; one needs to define a cost function $\mathcal{C}$ and a transformation model for $\mathrm{T}$ and one needs to select an optimization algorithm to perform the minimization of Eq. 1.

\subsection{Registration approach}

We apply a multi-stage registration approach. First, inter-subject CTA images are registered using an affine registration, to roughly align the two images. The Mean Square Difference (MSD) cost function :

$$
M S D\left(\mathcal{C}, I_{F}, I_{M}\right)=\frac{1}{\left|\Omega_{F}\right|} \sum_{x_{i} \in \Omega_{F}}\left[I_{F}\left(x_{i}\right)-I_{M}\left(T_{\mathcal{C}}\left(x_{i}\right)\right)\right]^{2}
$$

is calculated using 2048 image samples, randomly chosen in each iteration in the entire image domain, and 256 iteration of a stochastic gradient descent optimizer are performed ${ }^{8}$.

The affine registration result is then used as an initialization for a B-spline (non-rigid) registration. A four resolutions coarse-to-fine sampling strategy is performed. At each resolution level, 2048 iterations are executed. In each iteration, one voxel is selected randomly in the entire image domain; the remaining images samples (2047) are picked in a 50-mm square neighborhood around that voxel. The B-spline grid is defined by control points with 20-mm separation while the algorithm optimizes the Mutual Information (MI) localized similarity measure. We used the following MI definition given by Thevenaz and Unser ${ }^{9}$ :

$$
M I\left(\mathcal{C}, I_{F}, I_{M}\right)=\sum_{m \in L_{M}} \sum_{f \in L_{F}}\left(p(f, m ; \mathcal{C}) \log _{2}\left(\frac{p(f, m ; \mathcal{C})}{p_{F}(f) p_{M}(m ; \mathcal{C})}\right)\right)
$$

where $L_{F}$ and $L_{M}$ are sets of regularly spaced intensity bin centers, $p$ is the discrete joint probability, and $p_{F}$ and $p_{M}$ are the marginal discrete probabilities of the fixed and moving image, obtain by summing $p$ over $m$ and $f$, respectively.

In both steps, a random coordinate sampler is used, which is not limited to voxel positions: coordinates between voxel positions can also be selected. A $3^{\text {rd }}$-order B-spline interpolator is used to derive values $I_{M}\left(T_{\mathcal{C}}\left(x_{i}\right)\right)$ of the moving image. 


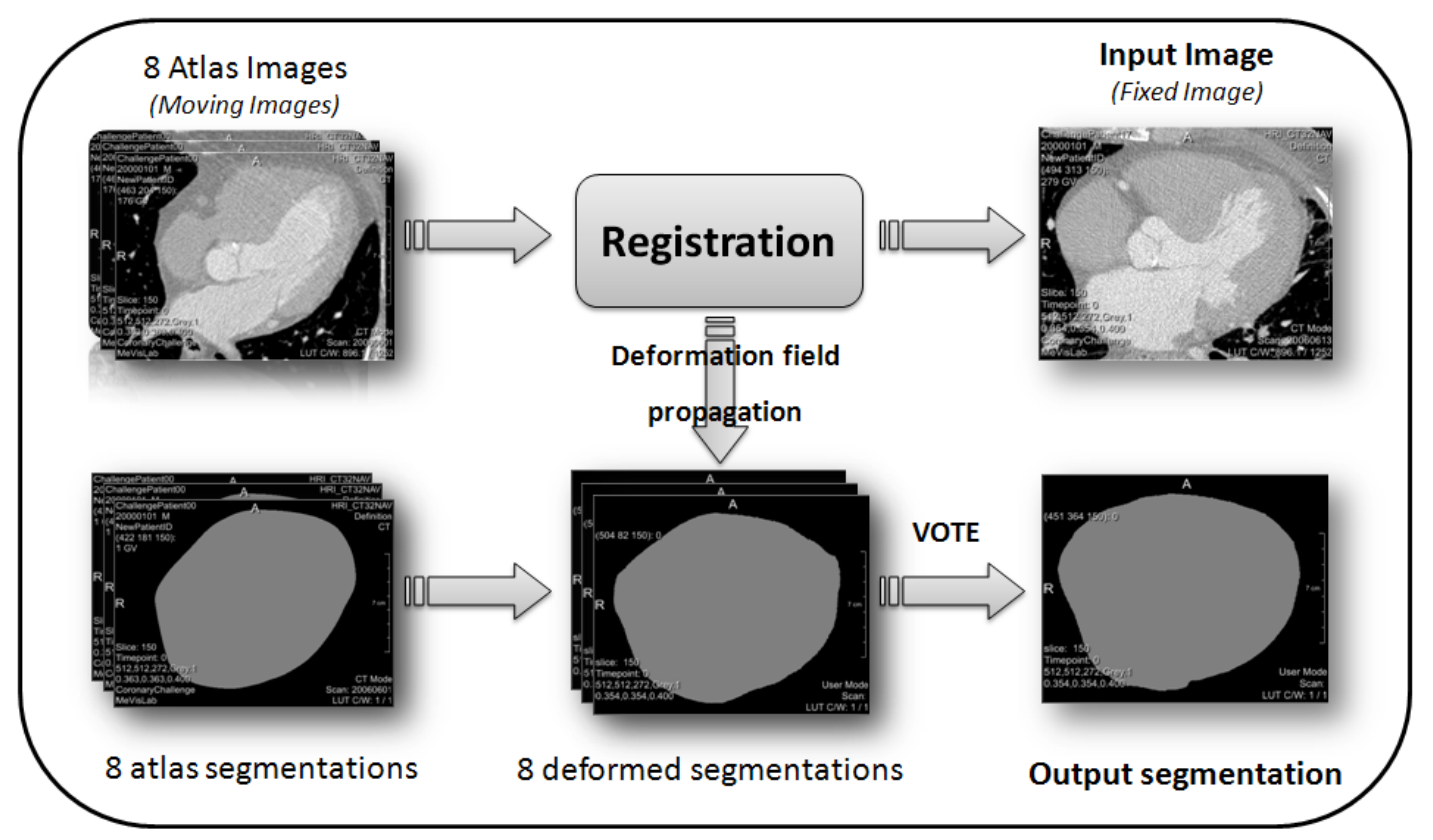

Figure 1. Multi-atlas based segmentation

We performed the registration using Elastix ${ }^{10}$, a package for medical image registration, publicly available on http: //elastix.isi.uu.nl/, and based on the Insight Segmentation Toolkit ITK (http://www.itk.org).

These registration parameters were determined with an 8 atlas images leave-one-patient-out pilot experiment, where we optimized the Dice coefficient of the cardiac chambers segmentations, and were used in all the experiments outlined in this paper.

\subsection{Multi-Atlas Based segmentation}

Multi-atlas based segmentation requires a set of $\mathrm{N}$ images, the atlas images $I_{M_{1, \ldots N N}}$, and their $\mathrm{N}$ corresponding (often manually) labeled images $L_{M_{1}, \ldots, N}$. The segmentation of an image $I_{F}$ is executed in three steps. Firstly, $I_{F}$ is registered with each of the moving atlas images $I_{M_{1, \ldots, N}}$, resulting in $N$ transformations $\widehat{T}_{1, \ldots, N}$. Then, the transformation $\widehat{T}_{i}$ is applied to $L_{M_{i}}, \forall i \in[1 \ldots N]$. This results in segmentations $L_{F_{i}}=\widehat{T}_{i}\left(L_{M_{i}}\right)$ of the fixed image $I_{F_{i}}$. Finally, a decision fusion algorithm is used to combine these $N$ segmentations into a single segmentation $L_{F}$. In our work, we used Majority Voting (MV).

\section{MATERIALS}

\subsection{Data}

251 CTA datasets were acquired in the Erasmus MC, University Medical Center Rotterdam, The Netherlands.

These 251 datasets contain the 32 CTA datasets that were used in the Rotterdam Coronary Artery Algorithm Evaluation Framework $^{11}$, which were randomly selected from a series of patients who underwent a cardiac CTA examination between June 2005 and June 2006.

The image quality (poor, moderate, good) and presence of calcium (low, moderate, high) for each of these 32 datasets was visually assessed by a radiologist with three years experience in cardiac CT. Based on these scorings, a representative group of 8 training CTA datasets to be used as atlas images was selected from these 32 datasets. The remaining 243 datasets were used as testing data for the qualitative evaluation.

Twenty datasets were acquired with a 64-slice CT scanner and twelve datasets with a dual-source CT scanner (Sensation 64 and Somatom Definition, Siemens Medical Solutions, Forchheim, Germany). Diastolic reconstructions were used, with 

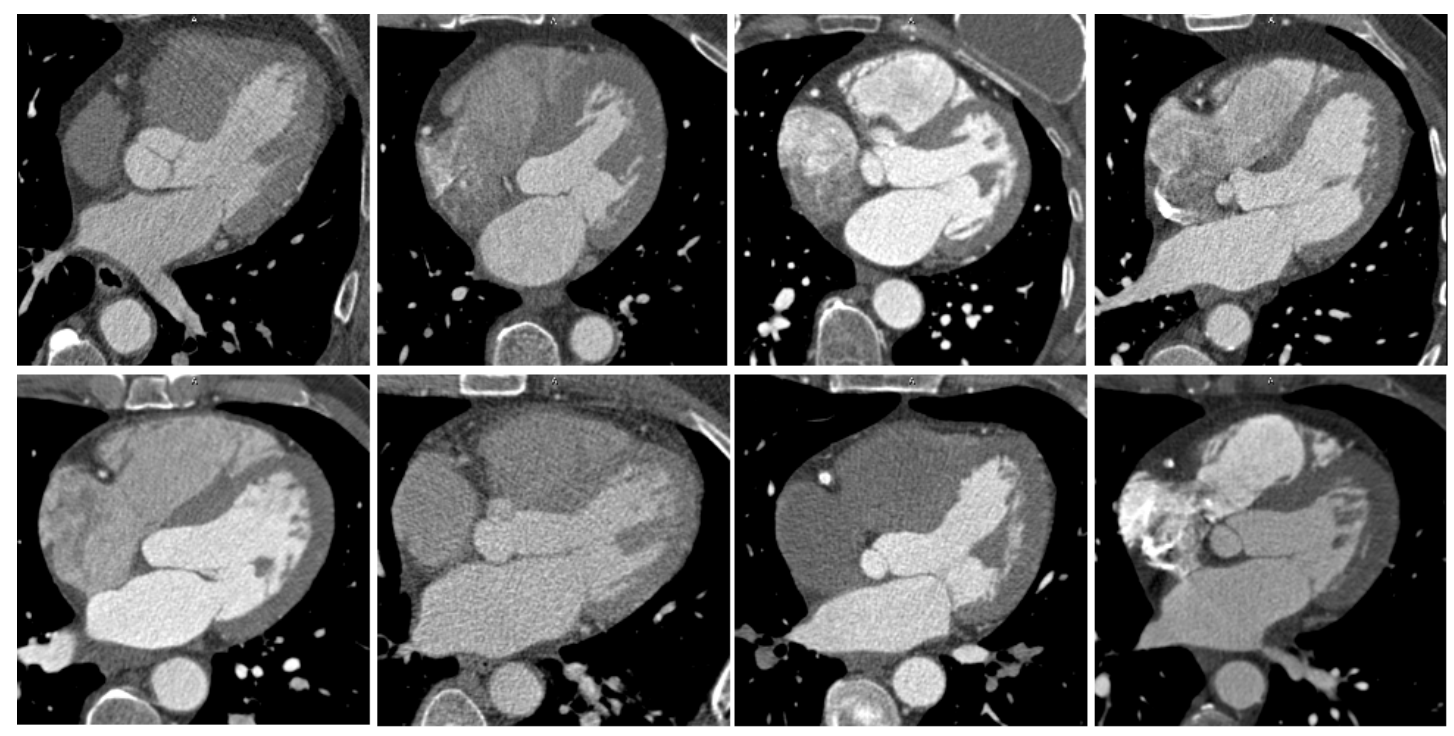

Figure 2. Eight atlas images - Variation of the image quality and presence of calcium in each dataset

reconstruction intervals varying from $250 \mathrm{~ms}$ to $400 \mathrm{~ms}$ before the R-peak. Three datasets were reconstructed using a sharp (B46f) kernel, all others were reconstructed using a medium-to-smooth (B30f) kernel. The mean voxel size of the datasets was $0.32 \times 0.32 \times 0.4 \mathrm{~mm}^{3}$.

\subsection{Manual segmentation}

The cardiac atlas was created by student manual labeling (Obs.1) of the 8 training CTA datasets, followed by a correction step performed by a radiologist. In the remainder of this paper, we refer to this data as the reference standard. Following the same segmentation guidelines (cardiac anatomical definition), a second radiologist (Obs.2) annotated both the heart and left ventricle. The segmentations of the second radiologist were used to compare the performance of our method with inter-observer variability.

The manual annotations were performed on the three orthogonal view (axial, coronal \& sagital) of the 3-D CTA data, according to the following guidelines and the definition given by Boxt ${ }^{12}$ :

- The heart (Heart) is delimited by the parietal pericardium. The parietal pericardium may be identified as a paperthin high signal intensity surface surrounding the heart and great arteries. Direct visualization of the parietal pericardium depends upon the presence and extent of low-density fatty deposition in the pericardial fat pad and middle mediastinum.

- The aorta (Ao) includes the ascending aorta trunk, the right aortic sinus, the left aortic sinus and stops at the aortic valve.

- The left ventricle ( $\mathbf{L V})$ starts at the mitral valve, goes to the main LV cavity, then to the Left Ventricle Outflow Track (LVOT), and stops at the aortic valve. The endocardium LV (endoLV) contours are matching the outer surface of the LV. The epicardium LV (epiLV) contours are matching the inner part of the LV, excluding the papillary muscles of the segmentation.

- The right ventricle (RV) starts at the tricuspid valve, then goes to the RV main cavity, to the Right Ventricle Outflow Track (RVOT), and stops at the pulmonary valves, excluding the pulmonary trunk.

- The left atrium (LA) starts at the pulmonary veins, excluding the branches (high inter-patient location variability) so that a contour is drawn through the veins. It includes the main LA cavity, and stop at the mitral valve. Left Atrium Appendage (LAA) is included in the segmentation. 
- The right atrium (RA) includes the right atrium appendage (RAA) and the main RA cavity. Both the inferior and superior vena cava are excluded, like the coronary sinus (CS).

Manual annotation of all the cardiac chambers was very time consuming and took 6 to 8 hours per dataset, depending on the image quality.

\section{EXPERIMENTS \& RESULTS}

\subsection{Quantitative evaluation : 8 cases leave-one-patient-out test}

We quantitatively evaluated our method using the 8 manually annotated atlas images with a leave-one-patient-out test. All the 8 atlas images were segmented with the proposed method using the seven remaining images as an atlas. Multiple metrics for segmentation performance are commonly used. In our evaluation we used the Dice Similarity Coefficient:

$$
D S C=\frac{2(M \cap S)}{M+S}
$$

the Tanimoto Coefficient (TC):

$$
T C=\frac{D S C}{2-D S C}
$$

and the mean shortest Euclidean distance, between automatic surface $\left(S_{i}\right)$ and reference standard $\left(M_{i}\right)$ :

$$
\varepsilon=\sqrt{\frac{1}{N} \sum_{i=1}^{N}\left(S_{i}-M_{i}\right)^{2}}
$$

Table 1 shows the mean shortest Euclidean distance and standard deviation between the automatic and reference standard (Auto. vs. Obs1), between the automatic and radiologist segmentations (Auto. vs. Obs2), and finally the inter-observer variability (Obs.1 vs. Obs2). It highlights that the error between the automatic and the reference standard is either close to (heart) or smaller than(endoLV) the inter-observer variability. Generally segmentation errors occur at the valves and near the apex.

Furthermore, quantitative results of the fully-automatic cardiac segmentation are presented in Table 4, along with results reported by others. A mean segmentation error of $1.05 \pm 1.30 \mathrm{~mm}$ and an average Dice coefficient of 0.93 demonstrate the high accuracy of the proposed approach.

In order to reduce the computation time, we tested our method on both original data and down-sampled data $(1 \times 1 \times$ $1 \mathrm{~mm}^{3}$ voxel size). Experiments demonstrated that decreasing the image resolution had no effect on the accuracy. Consequently, in the qualitative evaluation, down-sampled data were used, decreasing the computation time to approximately 25 minutes for the complete four-chamber segmentation.

\subsection{Qualitative evaluation on 243 testing datasets}

The robustness of our method is evaluated by a large-scale qualitative validation: 243 additional cardiac CTA datasets were segmented with the atlas-based approach. For the qualitative evaluation, we used a segmentation quality grade classification, based on regional segmentation accuracy, as proposed by Abadi et al. ${ }^{1}$ (see Table 2).

Abadi performed segmentation of the cardiac structures using the model-based algorithm developed by Ecabert et $a l{ }^{3}$. We achieved the same classification on 243 CTA data. Our results are presented in Table 3. The quality of the segmentation was in most of the cases highly accurate (grade $1 \& 2$ ). While there was no complete segmentation failure (grade 5), we observed that errors occur at the apex, where the contrast with surrounding tissues is poor, at the valves and near the pulmonary artery bifurcations, whose shape and position vary significantly between patients. Overestimates are mainly due to inclusion of pericardial fat as a part of the myocardium. Figure 4 shows examples of resulting cardiac segmentations. 
Table 1. Cardiac Segmentation - 8 cases Leave-One-Patient-Out Approach - Mean Shortest Euclidean Distance \pm Standard Deviation (in millimeters) of automatic segmentation (Auto. vs. Obs1/Obs2) and inter-observer variability (Obs1 vs. Obs2) - The atlas images were manually segmented by Obs1.

\begin{tabular}{c|ccc|ccc|ccc}
\hline & & Auto. vs. Obs1 & $(\mathrm{mm})$ & \multicolumn{3}{c}{ Auto. vs. Obs2 } & $(\mathrm{mm})$ & \multicolumn{3}{c}{ Obs1 vs. Obs2 } & $(\mathrm{mm})$ \\
\hline Patient & Heart & epiLV & endoLV & Heart & epiLV & endoLV & Heart & epiLV & endoLV \\
\hline 000 & $1.53 \pm 1.92$ & $1.04 \pm 1.18$ & $0.91 \pm 1.02$ & $1.47 \pm 1.68$ & $1.02 \pm 1.04$ & $0.85 \pm 0.85$ & $0.95 \pm 1.20$ & $0.78 \pm 0.94$ & $1.63 \pm 1.42$ \\
001 & $1.45 \pm 2.20$ & $1.50 \pm 1.58$ & $0.62 \pm 0.70$ & $1.77 \pm 2.69$ & $0.92 \pm 1.00$ & $1.19 \pm 0.98$ & $0.68 \pm 1.06$ & $1.08 \pm 1.05$ & $1.16 \pm 0.13$ \\
002 & $1.19 \pm 1.30$ & $0.85 \pm 0.95$ & $0.61 \pm 0.67$ & $1.22 \pm 1.43$ & $1.00 \pm 1.17$ & $0.91 \pm 0.88$ & $0.48 \pm 0.68$ & $0.62 \pm 0.69$ & $0.74 \pm 0.71$ \\
003 & $1.29 \pm 1.59$ & $0.84 \pm 1.00$ & $0.79 \pm 1.00$ & $1.21 \pm 1.58$ & $0.86 \pm 1.02$ & $0.78 \pm 0.97$ & $0.73 \pm 0.96$ & $0.55 \pm 0.71$ & $0.99 \pm 0.93$ \\
004 & $0.68 \pm 0.81$ & $1.26 \pm 1.1 .33$ & $0.61 \pm 0.77$ & $0.87 \pm 1.66$ & $1.17 \pm 1.34$ & $0.73 \pm 0.83$ & $0.72 \pm 1.52$ & $0.96 \pm 1.27$ & $0.58 \pm 0.60$ \\
005 & $1.50 \pm 2.30$ & $0.94 \pm 1.15$ & $0.61 \pm 0.65$ & $1.79 \pm 3.05$ & $1.12 \pm 1.24$ & $0.57 \pm 0.53$ & $1.24 \pm 2.02$ & $0.74 \pm 0.89$ & $0.68 \pm 0.74$ \\
006 & $0.83 \pm 1.05$ & $1.20 \pm 1.21$ & $0.74 \pm 0.85$ & $0.88 \pm 1.25$ & $1.10 \pm 1.36$ & $0.84 \pm 0.81$ & $0.59 \pm 0.77$ & $0.71 \pm 0.77$ & $0.75 \pm 0.70$ \\
007 & $1.59 \pm 2.69$ & $0.60 \pm 0.74$ & $0.40 \pm 0.50$ & $0.59 \pm 0.77$ & $0.65 \pm 0.80$ & $0.80 \pm 0.83$ & $1.05 \pm 1.68$ & $0.57 \pm 0.87$ & $0.62 \pm 0.67$ \\
\hline Mean & $1.27 \pm 1.73$ & $1.03 \pm 1.14$ & $0.67 \pm 0.78$ & $1.39 \pm 2.13$ & $0.98 \pm 1.12$ & $0.83 \pm 0.84$ & $0.80 \pm 1.24$ & $0.75 \pm 0.90$ & $0.89 \pm 0.86$ \\
\hline
\end{tabular}

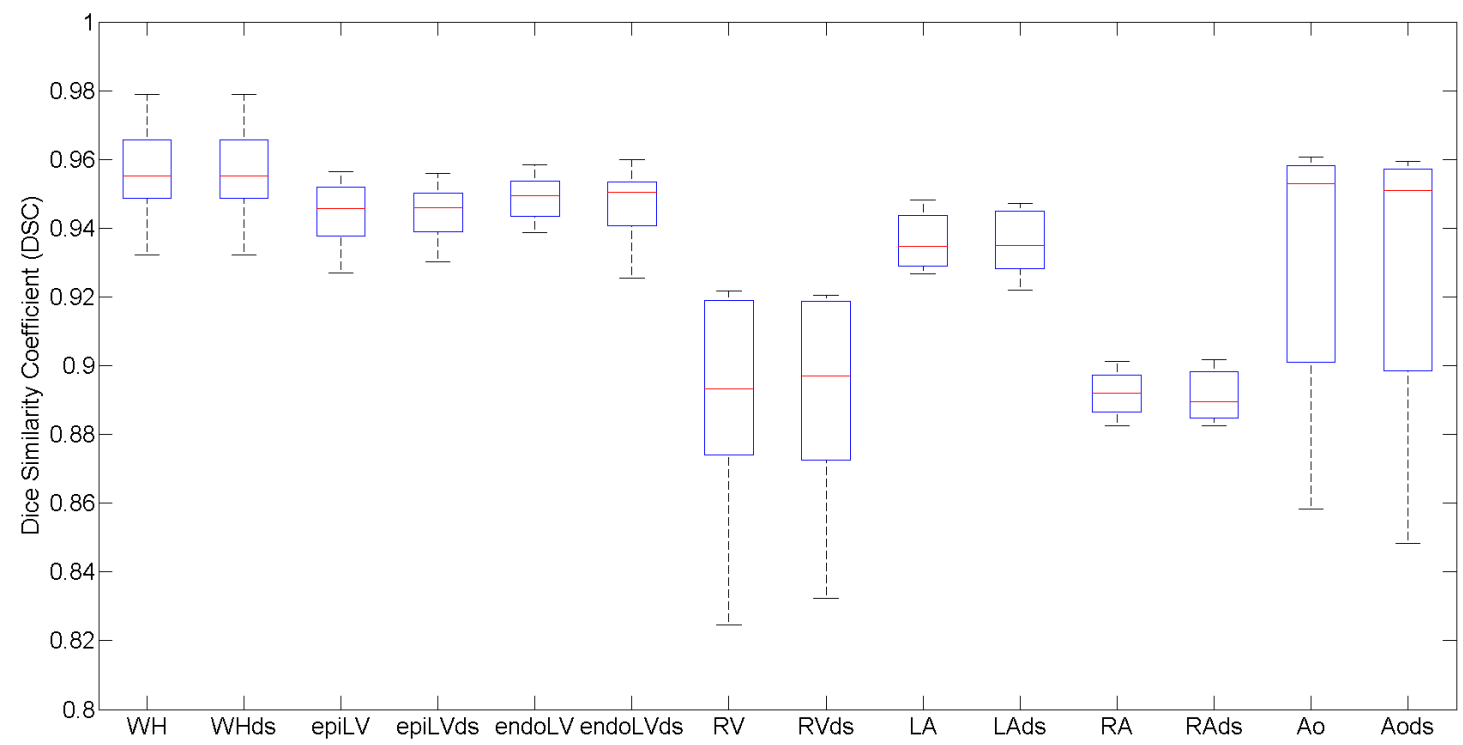

Figure 3. Cardiac Segmentation - 8 cases Leave-One-Patient-Out Approach - Dice Similarity Coefficient (DSC) - Atlas-based segmentation on original data $0.32 \times 0.32 \times 0.4 \mathrm{~mm}^{3} \&$ down-sampled data $1 \times 1 \times 1 \mathrm{~mm}^{3}$. From left to right, couples original/down-sampled: WH, epiLV, endoLV, RV, LA, RA and Ao

Table 2. Cardiac segmentation quality grade classification, published in Abadi et al. ${ }^{1}$

\begin{tabular}{ll}
\hline Grade & Description \\
\hline 1 & Very accurate: Deviation up to $1 \mathrm{~mm}$ \\
2 & Most regions accurate: $1-2$ regions may deviate up to $3 \mathrm{~mm}$ \\
3 & Most regions accurate: 1 region may deviate up to $1 \mathrm{~cm}$ or $>2$ regions may deviate up to $3 \mathrm{~mm}$ \\
4 & A significant region (up to $50 \%$ ) has not been segmented or has been incorectly segmented \\
5 & Segmentation failed
\end{tabular}


Table 3. Qualitative evaluation based on the grade classification outlined in Table 2 - Results in percentages - Our method was evaluated on 243 datasets while Ecabert et al. segmentation technique ${ }^{3}$ has been assessed on 100 datasets in the work of Abadi et al. ${ }^{1}$

\begin{tabular}{l|cccccc|ccccc}
\hline & \multicolumn{7}{c|}{ Our method } & \multicolumn{4}{c}{ Ecabert et al. ${ }^{13}$} \\
\hline Grade & Heart & epiLV & endoLV & RV & LA & RA & epiLV & endoLV & RV & LA & RA \\
\hline 1 & $51 \%$ & $14 \%$ & $62 \%$ & $36 \%$ & $46 \%$ & $40 \%$ & $3 \%$ & $35 \%$ & $68 \%$ & $49 \%$ & $53 \%$ \\
2 & $31 \%$ & $56 \%$ & $32 \%$ & $42 \%$ & $33 \%$ & $41 \%$ & $43 \%$ & $31 \%$ & $28 \%$ & $40 \%$ & $35 \%$ \\
3 & $14 \%$ & $28 \%$ & $5 \%$ & $18 \%$ & $17 \%$ & $18 \%$ & $54 \%$ & $34 \%$ & $2 \%$ & $8 \%$ & $6 \%$ \\
4 & $4 \%$ & $2 \%$ & $1 \%$ & $4 \%$ & $2 \%$ & $1 \%$ & $0 \%$ & $0 \%$ & $0 \%$ & $0 \%$ & $3 \%$ \\
5 & $0 \%$ & $0 \%$ & $0 \%$ & $0 \%$ & $0 \%$ & $0 \%$ & $0 \%$ & $0 \%$ & $2 \%$ & $3 \%$ & $3 \%$ \\
\hline
\end{tabular}

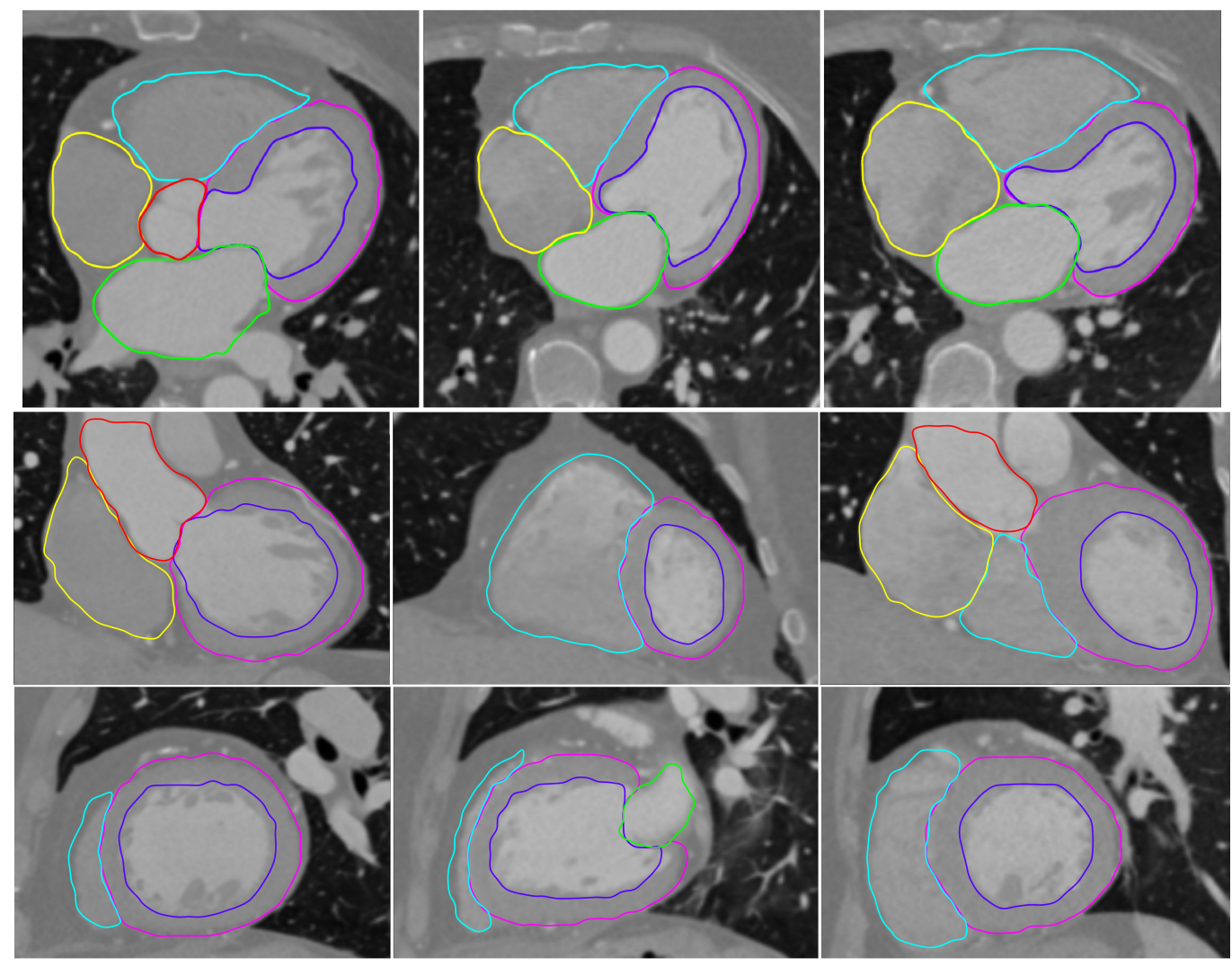

Figure 4. Fully automatic segmentation results from three patients out of the 243 testing down-sampled CTA datasets $\left(1 \times 1 \times 1 \mathrm{~mm}^{3}\right.$ voxel size)- Axial view (top), coronal view (middle) \& sagital view (bottom) - Segmentations: epiLV (pink), endoLV (purple), RV (blue), LA (green), RA (yellow) \& Ao (red). 
Table 4. Cardiac Segmentation - N cases Leave-One-Patient-Out Approach - Mean Dice Similarity Coefficient (DSC), mean Tanimoto Coefficient (TC), Mean Shortest Euclidean Distance \pm Standard Deviation

\begin{tabular}{|c|c|c|c|c|c|c|c|c|}
\hline & Patients & Heart & EpiLV & EndoLV & $\mathrm{RV}$ & LA & RA & Ao \\
\hline Mean DSC - Our method & 8 & 0.9562 & 0.9443 & 0.9455 & 0.8899 & 0.9362 & 0.8834 & 0.9304 \\
\hline Mean TC-Our method & 8 & 0.9165 & 0.8946 & 0.8969 & 0.8031 & 0.8802 & 0.7921 & 0.8720 \\
\hline Mean TC - Isgum et al. ${ }^{7}$ & 15 & 0.8847 & - & - & - & - & - & - \\
\hline Mean error (mm) - Our method & 8 & $1.25 \pm 1.73$ & $1.03 \pm 1.14$ & $0.67 \pm 0.78$ & $1.53 \pm 1.64$ & $0.72 \pm 0.97$ & $1.49 \pm 1.78$ & $0.63 \pm 1.04$ \\
\hline Mean error (mm) - Funka-Lea et al. ${ }^{5}$ & 9 & $4.10 \pm 5.50$ & - & - & - & - & - & - \\
\hline Mean error (mm) - Ecabert et al..$^{3}$ & 28 & $0.82 \pm 1.00$ & $0.98 \pm 1.32$ & $0.82 \pm 1.07$ & $0.84 \pm 0.94$ & $0.71 \pm 0.88$ & $0.89 \pm 0.96$ & $0.74 \pm 0.89$ \\
\hline Mean error (mm) - Zheng et al. ${ }^{4}$ & 186 & - & $1.21 \pm 0.41$ & $1.13 \pm 0.55$ & $1.55 \pm 0.38$ & $1.32 \pm 0.42$ & $1.57 \pm 0.48$ & - \\
\hline
\end{tabular}

\section{DISCUSSION}

In Table $3 \& 4$, we compare the performances of our method with: 1) the graph-cuts based technique of FunkaLea et $a l .{ }^{5}$ 2) the atlas-based segmentation of Isgum et al. ${ }^{7}$, 3) the model based approach of Ecabert et al. ${ }^{3}$, and 4$)$ the machine learning approach of Zheng et al. ${ }^{4}$. Quantitatively, our atlas-based segmentation performed better than three of the methods ${ }^{574}$ and as well as the fourth ${ }^{3}$. Qualitatively, our approach achieved better results for the LV segmentation, especially for the endocardium, while RV segmentation is in support of Ecabert's method. It should of course be noted that all evaluations have been performed on different datasets, thus care should be taking when comparing these studies. Only a comparative study based on a unique set of training and testing data would allow an objective evaluation of different cardiac segmentation methods.

For clinical application, computation time is one of the most important issues that has to be taken into account. Multiatlas based segmentation can be very time consuming, especially when using a high number of atlases. However, increasing the number of atlases does not necessarily increase performance. ${ }^{13}$ For different applications, a different trade-off between computational costs, and accuracy. In previous works, for different applications, different number of atlases have been used, e.g. 20 were found to work well for MR brain segmentation ${ }^{13}$, and around 8 was shown to work well for heart segmentation from chest CT. ${ }^{14}$ Recently, an adaptive multi-atlas segmentation (AMAS) framework has been proposed ${ }^{14}$ to select automatically optimal atlases from a database. Experiments demonstrated that the same performance was achieved, compared to the standard multi-atlas method, with lower computational costs.

Beside the computational costs, there are still a number of steps that could be taken to further improve our method. First, the atlas sharpness has an important effect on the segmentation accuracy. In our study, manual contours were converted to $3 \mathrm{D}$ binary masks, thus we did not achieve sub-voxel accuracy. Second, majority voting (VOTE) decision fusion may yield irregular shapes to be segmented. Improving both the atlas images and the decision fusion is likely to further improve results. Finally, accuracy improvements may also be achieved by combining the atlas-based segmentation with the result of a voxel classification method ${ }^{15}$.

\section{CONCLUSION}

In this paper, we have presented a fully automatic method for whole heart and cardiac chamber segmentation, on CTA data routinely acquired for diagnosis of the coronaries, using a multi-atlas based approach. High accuracy and robustness were demonstrated by a quantitative ( 8 cases) and large-scale ( 243 cases) qualitative evaluation of the method. The method can be used to provide additional diagnostic information in the assessment of CAD, without the need of an additional scan. 


\section{REFERENCES}

[1] Abadi, S., Roguin, A., Engel, A., and Lessick, J., "Feasibility of automatic assessment of four-chamber cardiac function with MDCT: Initial clinical application and validation," Eur J Radiol. , in press (2009).

[2] Lorenz, C. and von Berg, J., "A comprehensive shape model of the heart," Medical Image Analysis 10, 657-670 (2006).

[3] Ecabert, O., Peters, J., Schramm, H., Lorenz, C., von Berg, J., Walker, M. J., Vembar, M., Olszewski, M. E., Subramanyan, K., Lavi, G., and Weese, J., "Automatic model-based segmentation of the heart in CT images," IEEE Transaction on Medical Imaging 27, 1189-1201 (2008).

[4] Zheng, Y., Barbu, A., Georgescu, B., Scheuering, M., and Comaniciu, D., "Four-chamber heart modeling and automatic segmentation for 3D cardiac CT volumes using marginal space learning and steerable features," IEEE Transaction on Medical Imaging 27, 1668-1681 (2008).

[5] Funka-Lea, G., Boykov, Y., Florin, C., Jolly, M. P., Moreau-Gobard, R., Ramaraj, R., and Rinck, D., "Automatic heart isolation for CT coronary visualization using graph-cuts," in [Proc. 3rd IEEE International Symposium on Biomedical Imaging: Nano to Macro], (2006).

[6] Frangi, A. F., Niessen, W. J., and Viergever, M. A., "Three-dimensional modeling for functional analysis of cardiac images, a review," IEEE Transaction on Medical Imaging 20, 2-5 (2001).

[7] Isgum, I., Staring, M., Rutten, A., Prokop, M., Viergever, M., and van Ginneken, B., "Multi-atlas-based segmentation with local decision fusion - application to cardiac and aortic segmentation in CT scans," IEEE Transactions on Medical Imaging 28, 1000-1010 (2009).

[8] Klein, S., Pluim, J. P. W., Staring, M., and Viergever, M. A., "Adaptive stochastic gradient descent optimisation for image registration," International Journal of Computer Vision 81, 227-239 (2009).

[9] Thevenaz, P. and Unser, M., "Optimization of mutual information for multiresolution image registration," IEEE Transaction on Image Processing 9, 2083-2099 (Dec. 2000).

[10] Klein, S., Staring, M., Murphy, K., Viergever, M. A., and Pluim, J. P. W., "elastix: a toolbox for intensity-based medical image registration," IEEE Transactions on Medical Imaging 29, 196-205 (2010).

[11] Schaap, M., Metz, C., van Walsum, T., van der Giessen, A., Weustink, A., Mollet, N., Bauer, C., Bogunovic', H., Castro, C., Deng, X., Dikici, E., O’Donnell, T., Frenay, M., Friman, O., Hoyos, M. H., Kitslaar, P., Krissian, K., Khnel, C., Luengo-Oroz, M. A., Orkisz, M., Smedby, ., Styner, M., Szymczak, A., Tek, H., Wang, C., Warfield, S. K., Zambal, S., Zhang, Y., Krestin, G. P., and Niessen, W., "Standardized evaluation methodology and reference database for evaluating coronary artery centerline extraction algorithms," Medical Image Analysis 13(5), 701-714 (2009).

[12] Boxt, L., "CT anatomy of the heart," The International Journal of Cardiovascular Imaging 21, 13-27 (2005).

[13] Aljabar, P., Heckemann, R., Hammers, A., Hajnal, J., and Rueckert, D., "Multi-atlas based segmentation of brain images: Atlas selection and its effect on accuracy," NeuroImage 46(3), 726-738 (2009).

[14] van Rikxoort, E., Isgum, I., Arzhaeva, Y., Staring, M., Klein, S., Viergever, M., Pluim, J., and van Ginneken, B., "Adaptive Local Multi-Atlas Segmentation: Application to the Heart and the Caudate Nucleus," Medical Image Analysis 14, 39-49 (February 2009).

[15] van der Lijn, F., den Heijer, T., Breteler, M. M. B., and Niessen, W., "Hippocampus segmentation in MR images using atlas registration, voxel classification, and graph cuts," NeuroImage 43, 708-720 (2008). 